\title{
Calculation of production and decay of radioisotopes for future irradiation experiments and ion beam facilities
}

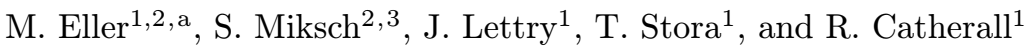 \\ 1 CERN, European Organization for Nuclear Research, Geneva, Switzerland \\ 2 Institute of Software Technology and Interactive Systems, Vienna University of Technology, Vienna, Autria \\ 3 Danube University, Krems, Austria
}

Received: January 31, 2007

\begin{abstract}
The design of future radioactive ion beam (RIB) facilites requires the forecast of radio isotope inventory after irradiation. At CERN - ISOLDE, we developed a software that estimates the activity of irradiated materials as a function of time dedicated to radioactive waste management. This tool can also be used for licensing procedures, planning of irradiation experiments and the estimation of yields.
\end{abstract}

PACS. 28.52.Av Theory, design, and computerized simulation - 29.25.-t Particle sources and targets 29.50.+v Computer interfaces - 29.87.+g Nuclear data compilation

\section{Introduction}

There are different numerical codes which provide isotope production cross sections. They are based on semi empirical models (Sigma [1]) or on the description of particle target interactions based on physical models (Fluka [2], MCNPX [3]). We have implemented an iterative algorithm that uses the isotope cross sections from these codes to calculate isotope production and decay over time.

We present in addition an interactive graphical interface. It displays the results in an intuitive and simple way to get an overview of activation, isotope production and laboratory classifications over time.

\section{Calculation}

According to the decay formula, the algorithm (illustrated figure 1) calculates the number of isotopes $N_{i}(t)$ :

$$
\begin{aligned}
N_{i}(t+\Delta t)= & N_{i}(t) \cdot e^{-\lambda \cdot \Delta t}+\phi \sigma \cdot X \cdot \Delta t \\
& +N_{i M}(t) \cdot\left(1-e^{-\lambda_{M} \cdot \Delta t}\right)
\end{aligned}
$$

where $N_{i}$ is the quantity of a given isotope $i$ and $N_{i M}$ the quantity of its mother isotope. $\phi$ is the integrated proton flux, $X$ the target thickness, $\sigma$ the production cross section, $\lambda$ the isotope's decay probability, $\lambda_{M}$ the decay probability of the mother isotope and $\Delta t$ is the time interval.

The algorithm performs this calculation for the list of isotopes produced in the target or resulting from their

\footnotetext{
a e-mail: Martin.Eller@cern.ch
}

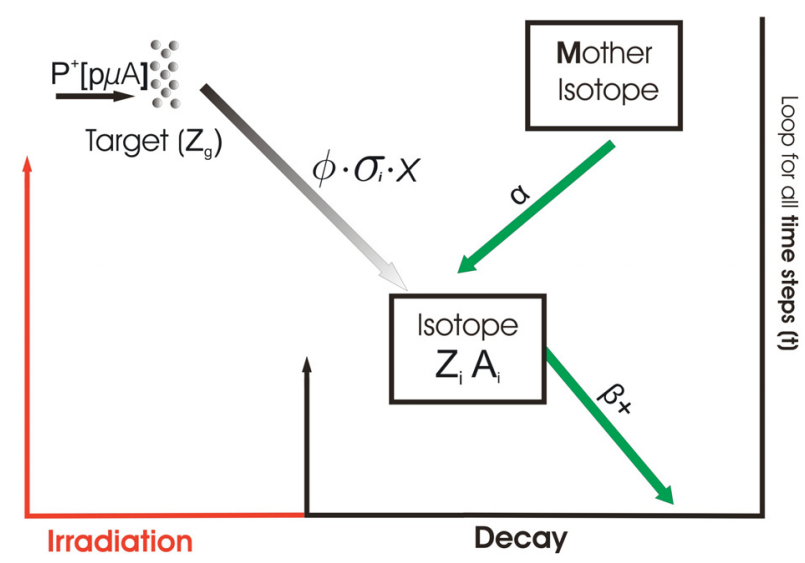

Fig. 1. The production of isotopes are taken from simulation codes. The algorithm runs a loop over all isotopes to calculate their decay and add the decayed fraction from the mother. The example shows the production of an isotope $Z_{i} A_{i}$. Its mother, via $\alpha$ decay, contributes to the quantity of the isotope $Z_{i} A_{i}$. The isotope itself decays via $\beta^{+}$emission. This iteration is performed for all isotopes. The smaller the iteration time steps, the higher the accuracy.

decay. E.g. in a Uranium target, irradiated by $1 \mathrm{GeV}$ protons, approximately 3500 isotopes are produced. The irradiation and decay is calculated according to user defined time steps. The decayed fraction is stored in a temporary array. This array is added to the corresponding daughter isotopes after the decay calculation of all elements in the list. A table of branching ratios and decay modes is used to obtain the proper daughter elements. Time steps between 


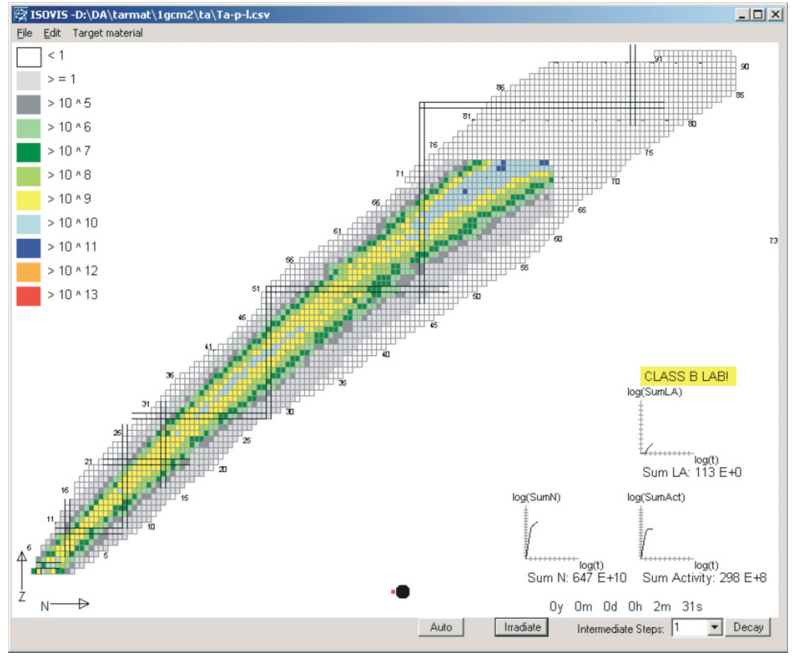

Fig. 2. Radioisotopic inventory of a Tantalum target. The target has been irradiated since 2.5 minutes by a proton beam with a current of $1 p \mu A$ and its thickness is $1 \mathrm{~g} / \mathrm{cm}^{2}$.

two calculation iterations are user defined and differ in irradiation and decay mode. During irradiation these time steps are kept constant.

After the irradiation phase only the decay is calculated and time steps follow a logarithmic time axis. The user sets the number of intermediate steps per order of magnitude. This is necessary to calculate target activations over long time spans once short lived isotopes have decayed. This is of particular interest for the estimation of activities for medium and long term storage.

The application takes following inputs:

Decay probabilities $\left(\lambda\left[s^{-1}\right]\right)$ : Are stored in a list of decay probabilities of known isotopes. It is used to calculate decays and activities.

Branching ratio and decay modes: Are used to determine the corresponding daughter elements.

Limits: Several lists of governmental or physical limits are used to calculate the dangerousness of an irradiated target. e.g. LA, the limits of Authorization of the Swiss government.

The application produces following outputs:

Quantity of radioisotopes: During irradiation and decay.

Activities: All the activities of the remaining isotopes.

Limits: A list of ratios between activity and authorization limits $\left(L a_{i}=\right.$ Activity $\left._{i} / L A_{i}\right)$ is stored for the remaining isotopes.

Pictures: All above mentioned values are used to draw a color coded nuclide chart with supportive graphs. These pictures can be stored or even be connected to a movie. (see figure 2).

\subsection{Simplifications schemes}

To keep the calculation time short (to run on standard personal computer) and simplify the algorithm some simplifications in the physics model were made:

- Only $\alpha$ and $\beta$ decays are taken into account.

- All produced isotopes are assumed to be in the ground state.

The result is an order of magnitude, which is sufficient for the desired goal.

\section{Graphical user interface}

The calculation algorithm is embedded in an application that is simple to use and that presents the results in a visual way. The software delivers easy to grasp visual representations of the isotope production, isotope inventory, target activation and safety levels (see figure 2).

The user can interact with the application to explore the data sets. Zooming and filtering provide interaction possibilities to see values in more details or to obtain an overview.

The results can be stored as pictures, comma separated files (csv) and as animations which show the evolution over time.

For more information about the visual representations and graphical user interface see [4].

\section{Conclusion}

The presented application provides quick estimations of radioisotopic inventories following irradiation and decay for RIB facilities and experiments. It takes cross section data from different simulation codes and calculates the production and decay over time. The iterative calculation algorithm contains chosen simplifications of the physics processes to keep the calculation time short.

Emphasis was put in producing a simple user interface and a visual output. The results are stored in pictures and in raw data files.

Future work will focus on providing interfaces to other simulation codes and on the verification of the calculation algorithm to the results of irradiation experiments.

\section{References}

1. R. Silberberg, C.H. Tsao, A.F. Barghouty, ApJ 501, 911 (1998)

2. A. Fasso, A. Ferrari et al., CHEP03, La Jolla, USA

3. MCNPX Webpage, Los Alamos National Lab, P.O. Box 1663, Los Alamos, NM 87545, USA (2005),

http://mcnpx.lanl.gov/

4. M. Eller, S. Miksch, J. Lettry, IEEE Proc. IV06 (2006), p. 95 